\title{
Hypoxia-induced autophagy promotes gemcitabine resistance in human bladder cancer cells through hypoxia-inducible factor $1 \alpha$ activation
}

\author{
XIAOYU YANG ${ }^{1,2}$, HUBIN YIN ${ }^{1,2}$, YUNZHI ZHANG ${ }^{2}$, XINYUAN LI $^{1,2}$, \\ HANG TONG $^{1}$, YIZHOU ZENG ${ }^{1}$, QUAN WANG ${ }^{1}$ and WEIYANG HE ${ }^{1}$ \\ ${ }^{1}$ Department of Urology, and ${ }^{2}$ Chongqing Key Laboratory of Molecular Oncology and Epigenetics, \\ The First Affiliated Hospital of Chongqing Medical University, Chongqing 400016, P.R. China
}

Received November 16, 2017; Accepted April 17, 2018

DOI: $10.3892 /$ ijo. 2018.4376

\begin{abstract}
Overcoming the chemoresistance of bladder cancer is a pivotal obstacle in clinical treatments. Hypoxia widely exists in solid tumors and has been demonstrated to contribute to chemoresistance through hypoxia-inducible factor $1 \alpha(\mathrm{HIF}-1 \alpha)$-mediated autophagy in several types of cancer. However, it is unclear whether HIF-1 $\alpha$-mediated autophagy and chemoresistance occur in bladder cancer. The present study demonstrated that HIF-1 $\alpha$ was overexpressed in 20 bladder cancer tissues compared with matched paracarcinoma tissues. Gemcitabine-induced apoptosis during hypoxia was significantly reduced compared with that observed under normoxic conditions. In addition, hypoxia activated autophagy and enhanced gemcitabineinduced autophagy. Combined treatment using gemcitabine and an autophagy inhibitor (3-methyladenine) under hypoxia significantly increased gemcitabine cytotoxicity. Furthermore, it was demonstrated that hypoxia-activated autophagy depended on the HIF-1 $\alpha / \mathrm{BCL} 2 /$ adenovirus E1B $19 \mathrm{kDa}$ protein-interacting protein 3 (BNIP3)/Beclin1 signaling pathway. Suppressing HIF-1 $\alpha$ inhibited autophagy, BNIP3 and Beclin1, as well as enhanced gemcitabineinduced apoptosis in bladder cancer cells under hypoxic conditions. Consequently, the results of the present study demonstrated that hypoxia-induced cytoprotective autophagy counteracted gemcitabine-induced apoptosis through increasing HIF-1 $\alpha$ expression. Therefore, targeting HIF-1 $\alpha$-associated pathways or autophagy in bladder cancer may be a successful strategy to enhance the sensitivity of bladder cancer chemotherapy.
\end{abstract}

Correspondence to: Dr Weiyang He, Department of Urology, The First Affiliated Hospital of Chongqing Medical University, 1 Youyi Road, Yuzhong, Chongqing 400016, P.R. China

E-mail: weiyang361@aliyun.com

Key words: hypoxia, autophagy, hypoxia-inducible factor $1 \alpha$, chemoresistance, bladder cancer

\section{Introduction}

Urinary bladder cancer has become one of the most common urological malignancies, and its incidence ranks eighth among all malignant types of cancer. It is estimated that 79,030 new cases of bladder cancer and 16,870 associated mortalities occurred in 2017 (1). Muscle-invasive bladder cancer patients are at a great risk of recurrence and metastasis subsequent to radical cystectomy. Furthermore, the 5-year survival of these patients is only $30-50 \%(2,3)$. Chemotherapy is a beneficial treatment for prolonging the survival time of patients at an advanced tumor stage. Gemcitabine, a cytosine analogue that inhibits DNA synthesis, has been widely used to treat bladder cancer, and gemcitabine in combination with other chemotherapeutic drugs has become the preferred standard treatment for advanced urothelial carcinoma (4-6). However, drug resistance leads to treatment failure in bladder cancer. Therefore, it is important to explore the underlying mechanism of gemcitabine resistance.

It has been reported that the tumor microenvironment is involved in the development of chemoresistance (7). Solid tumors are commonly exposed to a hypoxic environment due to their abnormal vascular system, treatment- or malignancyassociated anemia, and low levels of intra-tumor infusion. The resulting hypoxia becomes an important feature of the tumor microenvironment (8). The adaptive response of cells to hypoxia is achieved by altering the transcription of certain genes. Hypoxia-inducible factor 1 (HIF-1) is an important nuclear transcription factor that regulates hypoxia responses in cells. HIF-1 controls the expression of several downstream genes, which affect energy metabolism, glycolysis, angiogenesis, DNA repair, and the cell cycle progression. These genes not only control tumor proliferation and metastasis, but also promote chemotherapy resistance (9-11).

In hepatocellular carcinoma cells, autophagy is a protective mechanism involved in the resistance to chemotherapy under hypoxic conditions (12). Cell autophagy is a complex process involving lysosomal degradation of intracellularly damaged organelles and long-lived proteins, providing for the reuse of raw materials for cell survival under external conditions, such as hypoxia, a lack of growth factors or nutrient starvation 
conditions (13). It has been demonstrated that HIF-1 is involved in autophagy regulation (14). The downregulation of HIF-1 $\alpha$ suppresses autophagy and promotes anticancer agent-induced apoptosis (15).

Therefore, in order to validate the hypothesis that HIF-1 $\alpha$ mediates hypoxia-induced protective autophagy associated with gemcitabine resistance, the present study investigated the impact of hypoxia on cells treated with gemcitabine. Next, the study examined whether autophagy inhibition reversed the cytotoxicity of bladder cancer cells under hypoxia, and investigated the underlying mechanism of hypoxia-induced autophagy leading to gemcitabine resistance.

\section{Materials and methods}

Reagents and antibodies. The anti-light chain 3B (LC3B) antibody (1:1,000; Cat. no. L7543) was purchased from Sigma-Aldrich (Merck KGaA, Darmstadt, Germany), the anti-p62 antibody (1:2,000; Cat. no. 610497) was obtained from BD Biosciences (New York, NY, USA), and the anti-HIF-1 $\alpha$ antibody (1:1,000; Cat. no. 113642) was purchased from Abcam (Cambridge, MA, USA). Anti-poly(ADP-ribose) polymerase (PARP; 1:1,000; Cat. no. 95325), anti-cleaved caspase-3 (1:1,000; Cat. no. 9664S) and anti-Beclin1 antibodies (1:1,000; Cat. no. 3495S) were obtained from Cell Signaling Technology, Inc. (Danvers, MA, USA). An antibody against BCL2/adenovirus E1B $19 \mathrm{kDa}$ protein-interacting protein 3 (BNIP3;1:500; Cat. no. sc-56167) was obtained from Santa Cruz Biotechnology, Inc. (Dallas, TX, USA). Anti- $\beta$-actin antibody (1:1,000; Cat. no. Abm-0001) was from Zoonbio Biotechnology Co., Ltd. (Nanjing, China), while anti-rabbit (1:5,000; Cat. no. ASS1006)/mouse (1:5,000; Cat. no. ASS1021) secondary antibodies were purchased from Abgent (San Diego, CA, USA). 3-Methyladenine (3MA; $5 \mathrm{mM}$ ), chloroquine (CQ; $10 \mu \mathrm{M})$, gemcitabine and cell counting kit-8 (CCK-8) reagents were obtained from Selleck Chemicals (Houston, TX, USA). 3MA and CQ were both formulated in triple-distilled water. The bladder cancer cells were treated with 3MA or CQ for $1 \mathrm{~h}$ at room temperature prior to stimulation with hypoxia and GEM. RPMI-1640 medium was obtained from HyClone (GE Healthcare Life Sciences, Logan, UT, USA). Fetal bovine serum (FBS) and trypsin were purchased from Thermo Fisher Scientific, Inc. (Gibco, Waltham, MA, USA). The green fluorescent protein (GFP)-LC3 adenoviral vectors were acquired from Hanbio Biotechnology Co., Ltd. (Shanghai, China).

Tissue sample collection. A total of 20 paired specimens of bladder cancer and matched adjacent tissues were collected from the Department of Pathology, The First Affiliated Hospital of Chongqing Medical University. All specimens were histopathologically diagnosed. Among the patients, there were 16 males and 4 females, aged 50-80 years with a mean age of 66.5 years. They were admitted from January, 2013 to October, 2017. No patient received chemotherapy or radiotherapy prior to surgery. In total, 6 patients underwent transurethral resection of the bladder tumor (TURBT) and 14 patients underwent radical curative resection the bladder tumor (RC). Pathological grading and staging were determined according to the WHO 1973 criteria for grade and the 2002 TNM classification system $(16,17)$. The study was approved by the Ethics Committee of the First Affiliated Hospital of Chongqing Medical University, and written informed consent was obtained from the patients or the patients' families.

Cell lines and culture conditions. The human bladder cancer T24 cells were provided by the Chongqing Key Laboratory of Molecular Oncology and Epigenetics at the First Affiliated Hospital of Chongqing Medical University [Chongqing, China; the cells were originally obtained from the American Type Culture Collection (ATCC), Manassas, VA, USA]. The cells were maintained in RPMI-1640 medium supplemented with $10 \% \mathrm{FBS}, 0.1 \mathrm{mg} / \mathrm{ml}$ penicillin and $0.1 \mathrm{mg} / \mathrm{ml}$ streptomycin at $37^{\circ} \mathrm{C}$. In order to simulate the hypoxic environment, a hypoxia incubator (Sanyo Electric Co., Ltd., Osaka, Japan) with $1 \% \mathrm{O}_{2}$ at $37^{\circ} \mathrm{C}$ was used for cell cultures under hypoxic conditions, as previously described (18).

Cell viability assay. Cells were seeded in 96-well plates at $5 \times 10^{3}$ cells/well with $200 \mu \mathrm{l}$ medium for $24 \mathrm{~h}$ and then treated with gemcitabine at the indicated concentrations $(0,5,10,20$, $30,40,50$ and $60 \mu \mathrm{M})$ under hypoxic or normoxic conditions for a further $24 \mathrm{~h}$. In order to simulate a hypoxic environment, a hypoxic incubator (Sanyo Electric Co., Ltd.) with $1 \% \mathrm{O}_{2}$ at $37^{\circ} \mathrm{C}$ was used to expose the cells to hypoxia. Following incubation, $10 \mu \mathrm{l}$ CCK-8 and $100 \mu 1$ medium were added into each well, and incubated for $1 \mathrm{~h}$. The absorbance $(450 \mathrm{~nm})$ was measured by the Tecan Infinite F200/M200 type multifunction microplate reader (Tecan Group, Ltd., Männedorf, Switzerland). The viability percentage of the cells based on the optical density (OD) was calculated as follows: Cell viability $(\%)=($ Average OD value of the experimental group average OD value of blank group) / (average OD value of control group - average OD value of blank group) x 100\%. All experiments were conducted in triplicate. The half maximal inhibitory concentration $\left(\mathrm{IC}_{50}\right)$ was calculated using SPSS software (version 17.0; SPSS, Inc., Chicago, IL, USA).

Annexin V/propidium iodide (PI) assay. The cells were treated as follows: i) When the cell density reached $70-80 \%$, the cells were pre-treated with $3 \mathrm{MA}(5 \mathrm{mM})$ for $1 \mathrm{~h}$, and then cells were incubated under hypoxic or normoxic conditions for $24 \mathrm{~h}$ with or without gemcitabine $(5 \mu \mathrm{M})$ treatment, respectively. The specific groups were as follows: The normoxic control group, hypoxic group, hypoxia plus 3MA group, GEM group, GEM group under hypoxic conditions and gemcitabine combined with the 3MA group under hypoxic conditions. ii) When the cell density reached $40-50 \%$, HIF- $1 \alpha$ siRNA and NC siRNA were transfected into T 24 cells for $48 \mathrm{~h}$, and the cells were then incubated under hypoxic or normoxic conditions for $24 \mathrm{~h}$ with or without gemcitabine $(5 \mu \mathrm{M})$ treatment, respectively. The specific groups were as follows: The normoxic control group, hypoxia group, GEM group, GEM group under hypoxic conditions, GEM with NC siRNA group under hypoxic conditions and the GEM with siHIF-1 $\alpha$ group under hypoxic conditions. The cells were then trypsinized, washed with cold PBS 3 times, collected and then resuspended in $200 \mu \mathrm{l}$ Annexin V-FITC binding buffer, which included $5 \mu \mathrm{l}$ Annexin V-FITC and $10 \mu \mathrm{l}$ PI for $10 \mathrm{~min}$ in the dark. For this, the Annexin V-FITC and PI kit (Beyotime Institute of Biotechnology, Haimen, China) was used. Subsequently, all cells were stained for $10 \mathrm{~min}$ in 
the dark. Apoptotic cells were measured by flow cytometry (BD Biosciences, San Jose, CA, USA).

Electron microscopy. Subsequent to the indicated treatments, the cells were digested by trypsin and collected by centrifugation at $1,200 \times \mathrm{g}$ for $10 \mathrm{~min}$ at room temperature. The cells were sequentially fixed with $2.5 \%$ glutaraldehyde for $2 \mathrm{~h}$ and $1 \%$ osmium tetroxide for $1 \mathrm{~h}$, and then dehydrated with acetone and embedded in Epon resin (Sigma-Aldrich/Merck KGaA, Darmstadt, Germany) and the embedded material was then cut into 50-nm-thick sections using an ultramicrotome. Ultra-thin $(50 \mathrm{~nm})$ slices were double-stained with uranyl acetate and lead citrate (both from Sigma-Aldrich/Merck KGaA) for $15 \mathrm{~min}$ at room temperature. Subsequently, a transmission electron microscope (Hitachi-HTT7700; Hitachi, Ltd., Tokyo, Japan) was used to observe the autophagosomes.

Confocalmicroscopy.Bladdercancercellswereseeded(105/well) into confocal culture dishes (20 mm; NEST Biotechnology Co., Ltd., Wuxi, China) for $24 \mathrm{~h}$, and an adenovirus vector carrying GFP-LC3 (acquired from Hanbio Biotechnology Co., Ltd.) was transfected into the cells for $24 \mathrm{~h}$ according to the manufacturer's instructions. Subsequent to the indicated treatments, the cells were fixed in $4 \%$ formaldehyde at room temperature for 10 min and then washed with phosphate-buffered saline (PBS). The samples were observed under a confocal laser scanning microscope (Carl Zeiss-LSM700; Zeiss AG, Jena, Germany), and the numbers of GFP-LC3-positive puncta in each cell were counted by ImageJ software version 1.48 (National Institutes of Health, Bethesda, MD, USA).

Small interfering RNA (siRNA) transfection. The bladder cancer cells were transfected with the following: HIF-1 $\alpha$ siRNA, 5'-GCUGAUUUGUGAACCCAUUTT-3' (sense) and 5'-AAUGGGUUCACAAAUCAGCTT-3' (antisense); control siRNA, 5'-UUCUCCGAACGUGUCACGUTT-3' (sense) and 5'-ACGUGACACGUUCGGAGAATT-3' (antisense). Transfection reagents (siRNA-Mate; GenePharma Co., Ltd., Shanghai, China) and Opti-MEM (Invitrogen; Thermo Fisher Scientific, Inc.) were used based according to the manufacturer's instructions. Transfection was performed at room temperature for $10 \mathrm{~min}$. The knockdown efficiency was then measured by immunoblotting.

Immunoblotting. In order to extract total protein lysates, treated cells were lysed in radioimmunoprecipitation assay lysis buffer and 1\% phenylmethanesulfonyl fluoride (both from Beyotime Institute of Biotechnology) and centrifuged at $12,000 \mathrm{x} \mathrm{g}$ for $15 \mathrm{~min}$ at $4^{\circ} \mathrm{C}$. A bicinchoninic acid kit (Beyotime Institute of Biotechnology) was then used to assess the protein concentration. Identical amounts of protein $(40 \mu \mathrm{g})$ in each lane were separated with SDS-PAGE (12\% gels) and transferred onto polyvinylidene difluoride membranes. After blocking in 5\% fat-free milk in Tris-buffered saline/Tween-20 (TBST) for $1 \mathrm{~h}$, the bands were probed with various antibodies (mentioned above) at $4^{\circ} \mathrm{C}$ overnight. On the following day, the bands were washed three times with TBST for $10 \mathrm{~min}$, then incubated with the goat anti-rabbit/mouse secondary antibody for $1 \mathrm{~h}$, and washed again three times with TBST. Finally, the bands were exposed to an enhanced chemiluminescence detection reagent (EMD Millipore, Burlington, MA, USA) and images were captured using the Fusion Chemical Imaging System (Vilber Lourmat, Paris, France). The membranes were exposed to a gel imager and the expression of the target protein was visualized by detecting specific bands. ImageJ software version 1.48 was used to quantify each specific blot band.

Immunohistochemistry. Formalin-fixed and paraffin-embedded tissue sections were dehydrated with xylene, hydrated with ethanol, and then soaked in $0.01 \mathrm{~mol} / 1$ citrate buffer for $30 \mathrm{~min}$ at $96^{\circ} \mathrm{C}$ to conduct antigen repair. Next, $3 \%$ hydrogen peroxide was used to eliminate the endogenous peroxidase. The sections were then blocked with $10 \%$ goat serum at $37^{\circ} \mathrm{C}$ for $30 \mathrm{~min}$ and incubated with the anti-HIF-1 $\alpha$ primary antibody overnight at $4^{\circ} \mathrm{C}$. On the following day, the slides were incubated at $37^{\circ} \mathrm{C}$ for $30 \mathrm{~min}$, washed with PBS, and incubated with the horseradish peroxidase-conjugated secondary antibody (OriGene Technologies, Inc., Beijing, China) for $30 \mathrm{~min}$. The slides were then stain with DAB (OriGene Technologies, Inc.) for $\sim 1 \mathrm{~min}$, and the cell nucleus was stained with hematoxylin for $10 \mathrm{sec}$. PBS instead of the primary antibody was used as a negative control. Differential expression of HIF-1 $\alpha$ staining was scored using a semiquantitative grading system based on the staining intensity and extent. The HIF-1 staining extent was scored according to the percentage of positive staining cells as follows: 0 , positive cells of $\leq 5 \% ; 1,10-25 \%$ positive cells; $2,26-50 \%$ positive cells; $3,51-75 \%$ positive cells; and $4,>75 \%$ positive cells. Similarly, the HIF-1 staining intensity was scored as follows: 0 , no staining; 1, low staining; 2 , mild staining; and 3 , high staining. The final score was the sum of the two indicators, and was between 0 and 7. A score of $\geq 4$ was considered to indicate high HIF-1 $\alpha$ expression, while a score of $<4$ was defined as low HIF- $1 \alpha$ expression (19). The immunohistochemical staining level was assessed and scored by two independent pathologists, who were blinded to the origin of the tumor and paracarcinoma tissues.

Statistical analysis. At least three independent experiments were utilized to collect the quantitative data, and results are presented as the mean \pm standard deviation. SPSS software (version 17.0; SPSS, Inc.) was used for the statistical analyses. One-way analysis of variance with Fisher's Least Significant Difference post hoc test was applied to analyze the statistical differences among different treatment groups. The statistical difference between two groups was analyzed by the least significant difference t-test. $\mathrm{P}<0.05$ was considered to be an indicator of a statistically significant difference.

\section{Results}

Overexpression of HIF-1 $\alpha$ in tumor tissues of bladder cancer patients. To determine whether hypoxia was a general characteristic in tumor tissues, the present study tested the expression of the hypoxia-specific indicator HIF-1 $\alpha$ in 20 paired bladder cancer and adjacent non-cancerous tissues using immunohistochemistry. HIF-1 $\alpha$ was mostly detected in the cytoplasm, and the majority of the tumor samples had higher HIF-1 $\alpha$ expression as compared with that in the matched paracarcinoma tissues (Fig. 1A-C). The quantitative scores of HIF-1 $\alpha$ expression in tumor tissues were almost 5-fold greater compared with those of the paracarcinoma tissues ( 5.15 vs. 0.71 , respectively; 


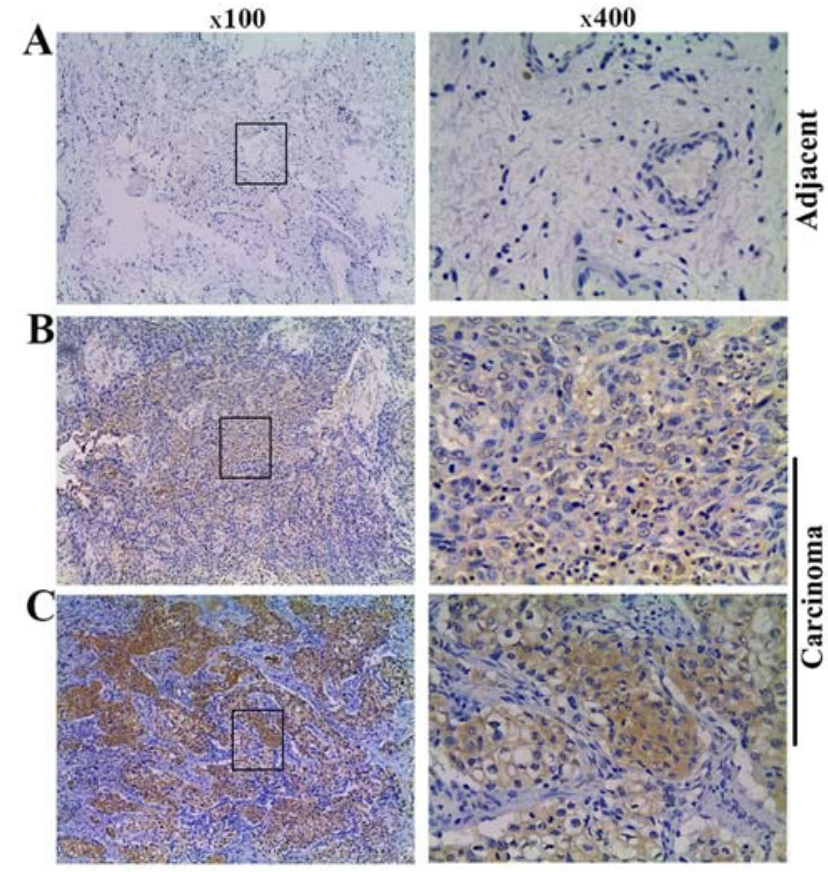

D

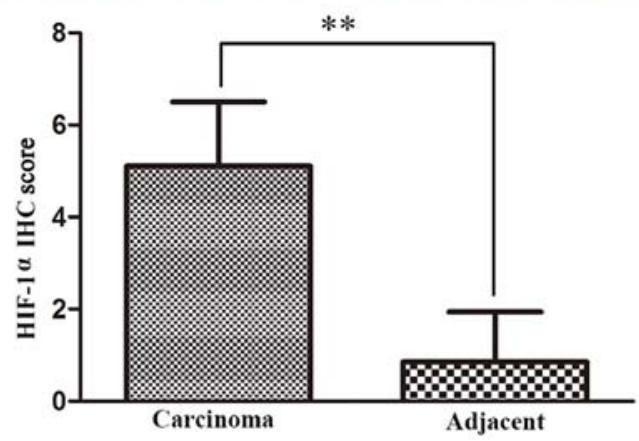

Figure 1. Increased expression of HIF-1 $\alpha$ in bladder cancer tissues compared with that in matched adjacent non-tumor tissues, as determined by immunohistochemical analysis. (A) Negative expression of HIF-1 $\alpha$ was observed in adjacent tissues (18/20 samples; magnification, x100). The right image shows the negative expression of HIF-1 $\alpha$ at a magnified view in the adjacent non-tumor tissues (magnification, $\mathrm{x} 400$ ). (B) Low expression (5/20 samples) and (C) high expression (15/20 samples) of HIF-1 $\alpha$ was observed in bladder tumor samples (magnification, $\mathrm{x} 100$ ). The right image shows the representative HIF-1 $\alpha$ expression at a magnified view in bladder cancer tissues (magnification, $x 400$ ). (D) Differences in HIF-1 $\alpha$ staining between cancer and adjacent tissues were scored using a semi-quantitative grading system, and the scores are shown in the diagram. ${ }^{* *} \mathrm{P}<0.01$. HIF-1 $\alpha$, hypoxia-inducible factor $1 \alpha$.

$\mathrm{P}<0.01$; Fig. 1D). These results revealed that hypoxia was a general characteristic in bladder cancer.

Hypoxia induces gemcitabine resistance in bladder cancer cells. To detect the impact of hypoxia on gemcitabine resistance, the chemosensitivity of the T24 cells was examined under hypoxic or normoxic conditions. The cells were exposed to a series of concentrations of gemcitabine ranging between 0 and $60 \mu \mathrm{M}$ for $24 \mathrm{~h}$ under normoxic conditions $\left(20 \% \mathrm{O}_{2}\right)$ or hypoxia $\left(1 \% \mathrm{O}_{2}\right)$. Subsequently, the CCK- 8 assay was used to evaluate the cell viability (Fig. 2A). The cell viability decreased in a dose-dependent manner and was higher conditions as compared with that under normoxic conditions. The half maximal inhibitory concentration $\left(\mathrm{IC}_{50}\right)$ value for $\mathrm{T} 24$ cells under normoxic conditions was $4.104 \pm 0.6132 \mu \mathrm{M}$, while the $\mathrm{IC}_{50}$ value of $\mathrm{T} 24$ cells conditions was $38.40 \pm 1.001 \mu \mathrm{M}$.
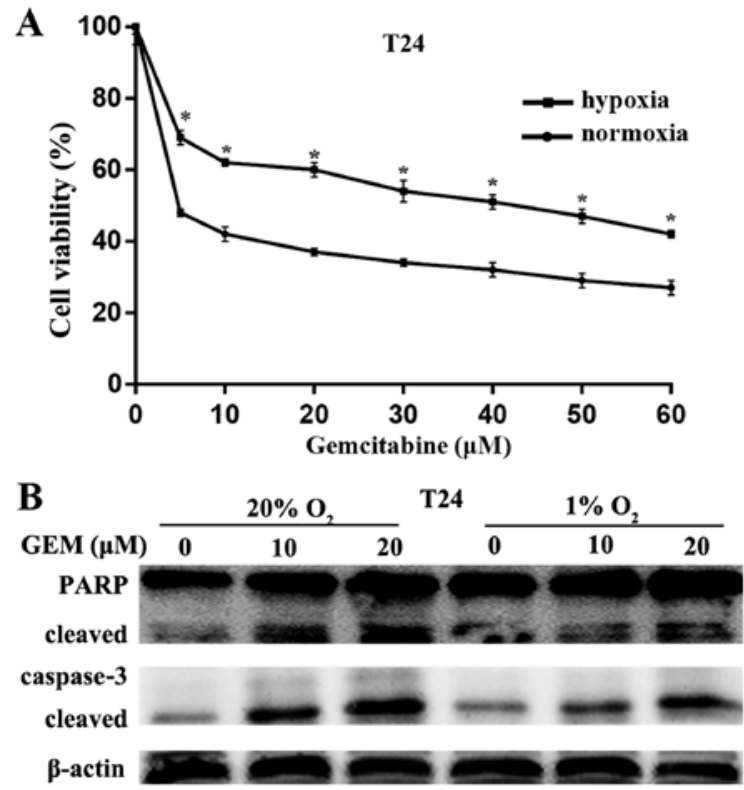

Figure 2. Bladder cancer cells are resistant to GEM under hypoxia. T24 cells were treated with GEM for $24 \mathrm{~h}$ under normoxia or hypoxia. (A) Cell viability was determined by a cell counting kit- 8 assay. Data are represented as the mean \pm standard deviation from three independent experiments. ${ }^{*} \mathrm{P}<0.05$. (B) PARP, cleaved PARP, caspase- 3 and cleaved caspase- 3 levels were measured by immunoblotting. GEM, gemcitabine; PARP, poly(ADP-ribose) polymerase.

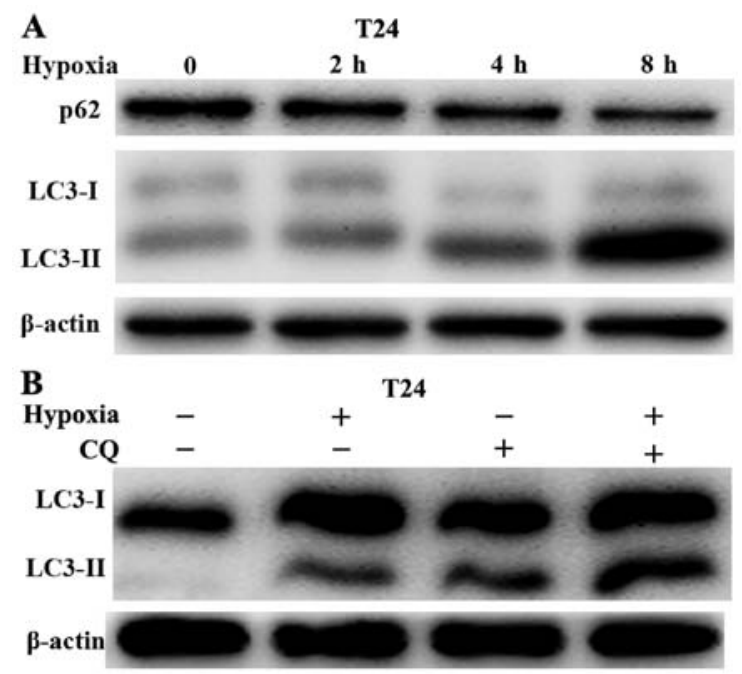

Figure 3. Hypoxia induced autophagy in bladder cancer cells. (A) T24 cells were cultured in complete medium under hypoxia for the indicated times. (B) T2 4 cells were pretreated with CQ $(10 \mu \mathrm{M})$ for $30 \mathrm{~min}$, and then incubated in complete medium under hypoxia or normoxia for an additional 8 h. p62, LC3-I and LC3-II protein levels were detected by immunoblotting. $\mathrm{CQ}$, chloroquine; LC3, light chain 3.

Therefore, the gemcitabine concentration of $5 \mu \mathrm{M}$ was utilized for the treatment of T24 cells in subsequent experiments. Furthermore, gemcitabine caused the cleaved caspase- 3 and cleavage of PARP in a dose-dependent manner (Fig. 2B). Under normoxic conditions, gemcitabine caused more significant cleaved caspase- 3 and cleavage of PARP as compared with that under hypoxia (Fig. 2B). These data suggested that hypoxia reduced the chemosensitivity of bladder cancer cells to gemcitabine. 
A

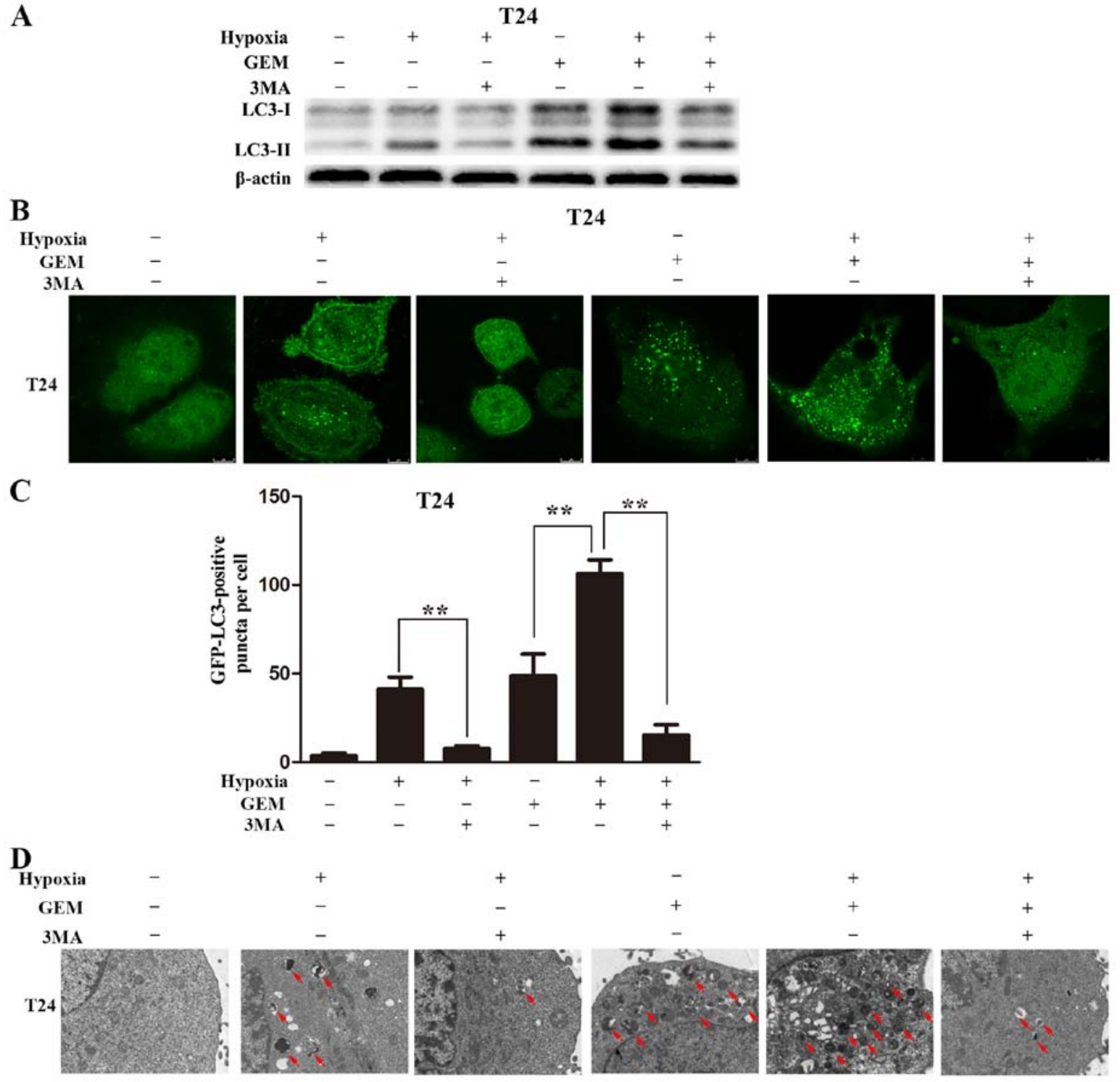

Figure 4. Hypoxia augmented GEM-induced autophagy in bladder cancer cells. (A) T24 cells were pretreated with 3MA (5 mM) for $1 \mathrm{~h}$, and then incubated in complete medium under hypoxia or normoxia with or without GEM $(5 \mu \mathrm{M})$ for an additional $8 \mathrm{~h}$. LC3 was detected by immunoblotting. (B) T24 cells were transfected with GFP-LC3 adenovirus for $24 \mathrm{~h}$, pretreated with 3MA $(5 \mathrm{mM})$ for $1 \mathrm{~h}$, and then incubated in complete medium under hypoxia or normoxia with or without GEM $(5 \mu \mathrm{M})$ for an additional $8 \mathrm{~h}$. GFP-LC3 puncta were detected by confocal microscopy (scale bar, $8 \mu \mathrm{m}$ ). (C) Number of GFP-LC3 puncta per cell (>10) were calculated, and are displayed in the histogram. (D) Cells were pretreated with 3MA (5 mM) for $1 \mathrm{~h}$, and then incubated in complete medium under hypoxia or normoxia with or without GEM $(5 \mu \mathrm{M})$ for an additional $8 \mathrm{~h}$. Autophagosomes were detected by transmission electron microscopy (scale bar, $2 \mu \mathrm{m})$. The red arrows designate the autophagosomes. Data represent the mean \pm standard deviation from three independent experiments. ${ }^{* *} \mathrm{P}<0.01$. GEM, gemcitabine; 3MA, 3-methyladenine; LC3, light chain 3.

Hypoxia induces autophagy in bladder cancer cells. Previous studies $(20,21)$ have demonstrated that hypoxia leads to resistance to gemcitabine, and it was further demonstrated that autophagy was induced during hypoxia and nutritional deficiency. Thus, the current study investigated whether hypoxia induced autophagy in bladder cancer cells. First, immunoblotting was used to confirm the occurrence of autophagy under hypoxic conditions. Hypoxia improved the expression of LC3-II, with p62 degradation occurring in a time-dependent manner (Fig. 3A). In addition, autophagy flux was examined following co-treatment with hypoxia and a lysosomal inhibitor, CQ $(10 \mu \mathrm{M})$. Although hypoxia or CQ alone increased LC3-II levels, the combination of hypoxia with CQ resulted in a marked increase in LC3-II levels (Fig. 3B). Taken together, these data revealed that hypoxia activated autophagy in bladder cancer cells.
Hypoxia elevates gemcitabine-induced autophagy in bladder cancer cells. The current study next examined the occurrence of autophagy in bladder cancer cells treated with gemcitabine under hypoxic conditions. T24 cells were incubated in complete medium under hypoxic or normoxic conditions, with or without gemcitabine for $8 \mathrm{~h}$. Exposure to hypoxia and treatment with gemcitabine increased LC3-II levels compared with the levels upon exposure to hypoxia or treatment with gemcitabine alone. In addition, the LC3-I to LC3-II conversion was almost completely abolished by the addition of 3MA $(5 \mathrm{mM})$ in the gemcitabine-treated cells under hypoxic conditions or in cells exposed to hypoxia alone (Fig. 4A).

Autophagy was also observed by monitoring the formation of GFP spots using confocal microscopy. Following transfection 

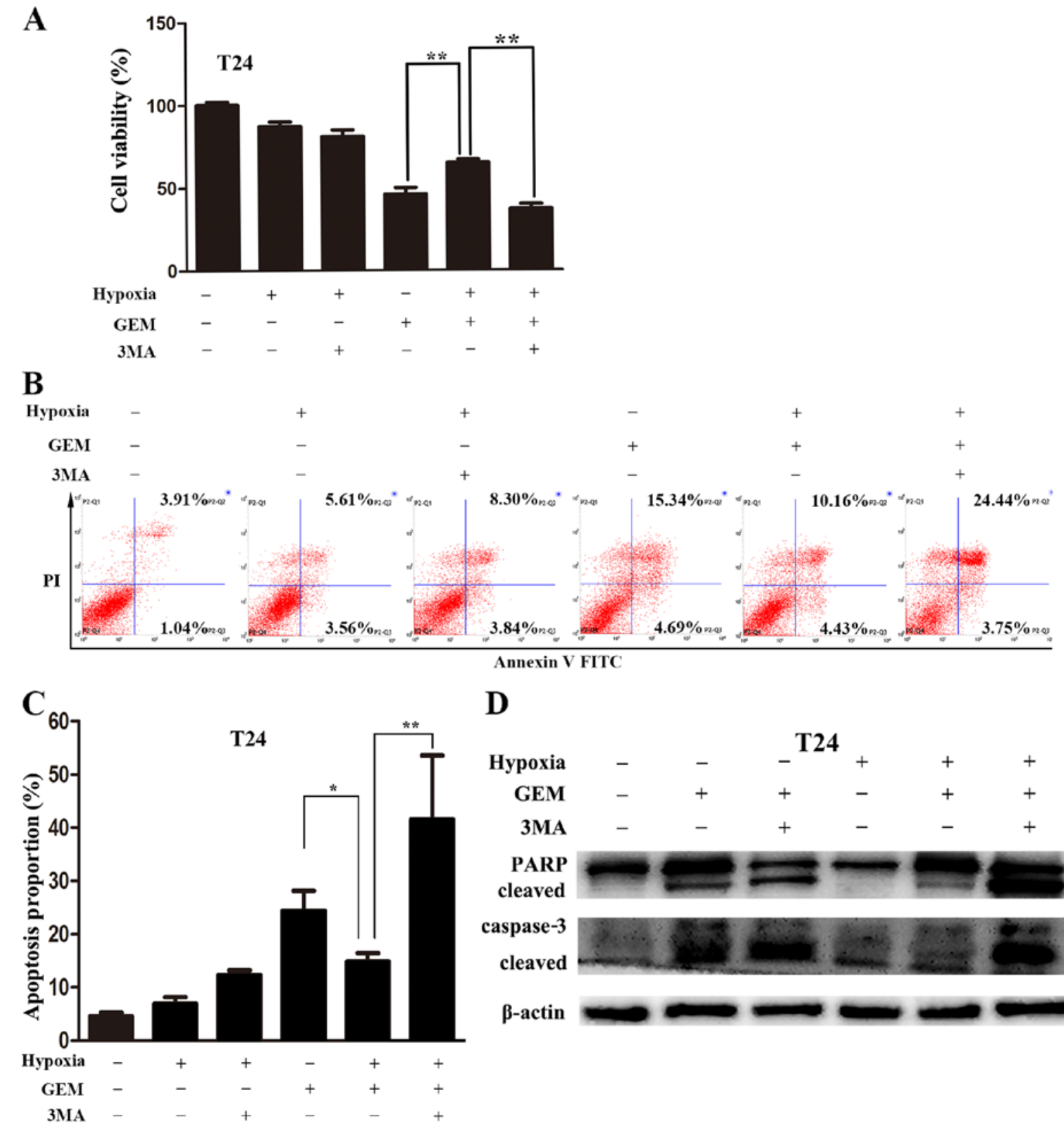

D
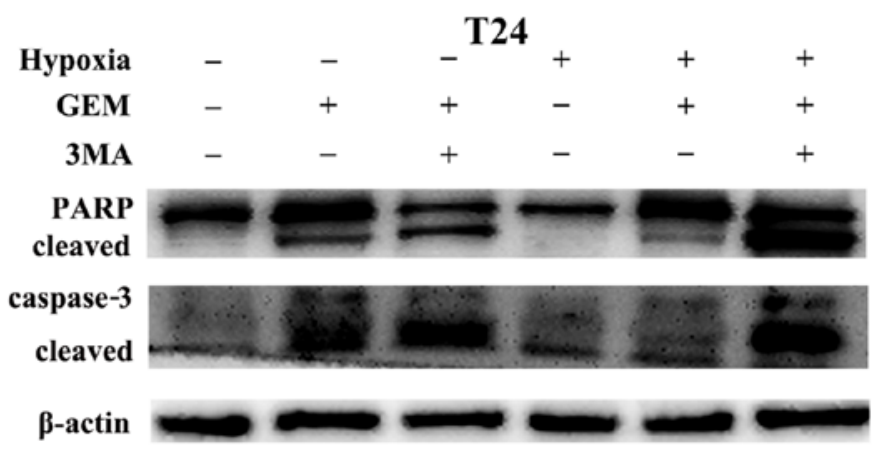

Figure 5. Inhibition of autophagy enhanced GEM-induced apoptosis under hypoxia. T24 cells were pretreated with 3MA (5 mM) for $1 \mathrm{~h}$, and then treated with or without GEM $(5 \mu \mathrm{M})$ for $24 \mathrm{~h}$ under hypoxia or normoxia. (A) Cell viability was detected by the cell counting kit-8 assay. (B) Apoptotic cell percentages were detected by flow cytometry. (C) Early apoptosis (Annexin $\mathrm{V}^{+} / \mathrm{PI}$ ) and late-stage apoptosis (Annexin $\mathrm{V}^{+} / \mathrm{PI}^{+}$) were calculated, and are shown in the histograms . (D) Cleaved caspase-3 and PARP were measured by immunoblotting. Data represent the mean \pm standard deviation from three independent experiments.

${ }^{* *} \mathrm{P}<0.01$ and " $\mathrm{P}<0.05$. GEM, gemcitabine; 3MA, 3-methyladenine; PARP, poly(ADP-ribose) polymerase; PI, propidium iodide.

using a GFP-LC3 adenovirus for $24 \mathrm{~h}$, the cells were maintained in complete medium under normoxic or hypoxic conditions, with or without gemcitabine for $8 \mathrm{~h}$. The number of GFP-LC3 puncta in the gemcitabine-treated cells under hypoxic conditions was significantly increased in comparison with that in the hypoxia or gemcitabine-treated cells alone. Furthermore, following pretreatment with 3MA, GFP-LC3 puncta accumulation significantly decreased in the gemcitabine-treated cells under hypoxic conditions or in cells cultured under hypoxic conditions alone (Fig. 4B and C).

Transmission electron microscopy was also used to observe autophagosomes. Autophagic vacuoles were detected following exposure to hypoxia or gemcitabine treatment alone, and the amount of autophagic vacuoles was augmented when cells were exposed to gemcitabine and hypoxia. However, following pretreatment with 3MA, the autophagic vacuoles decreased (Fig. 4D). In conclusion, these results indicated that hypoxia augmented gemcitabine-induced autophagy in bladder cancer cell lines.

Blocking autophagy enhances bladder cancer cell sensitivity to gemcitabine under hypoxic conditions. To verify that autophagy had a protective effect in bladder cancer cells obtaining resistance to gemcitabine under hypoxic conditions, 3MA, which is an inhibitor of phosphatidylinositol-3 kinase (PI3K), was utilized to inhibit autophagy. The CCK-8 assay revealed that 3MA $(5 \mathrm{mM})$ strongly enhanced the inhibitory effect of gemcitabine on cell viability under hypoxic conditions (Fig. 5A). 
A

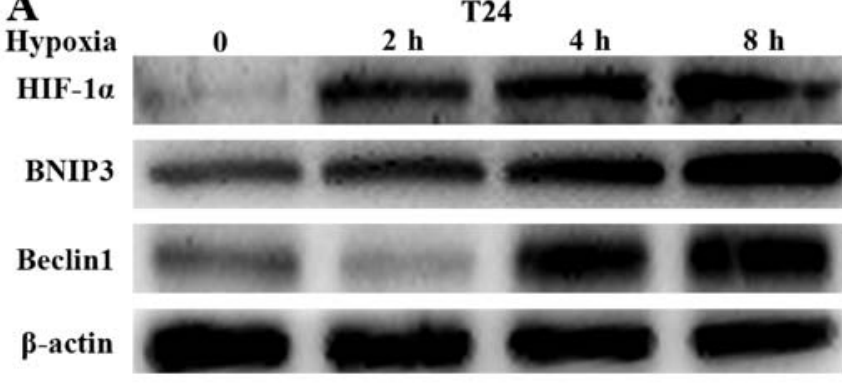

B
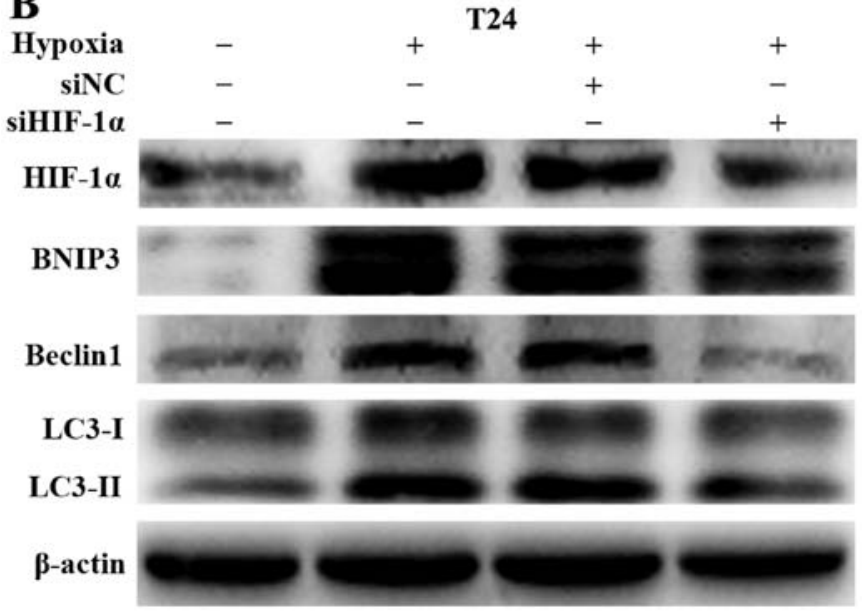

C
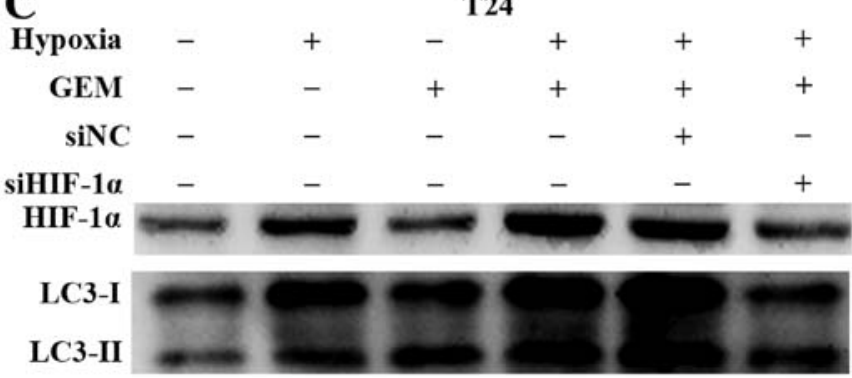

$\beta$-actin

Figure 6. HIF-1 $\alpha$ mediated hypoxia-induced autophagy. (A) T24 cells were cultured under hypoxia for the indicated times, and HIF-1 $\alpha$, BNIP 3 and Beclin1 levels were detected by immunoblotting. In addition, T24 cells were transfected with HIF-1 $\alpha$ siRNA or control siRNA for $48 \mathrm{~h}$, followed by (B) exposure to hypoxia for $8 \mathrm{~h}$ or (C) incubation with GEM (5 $\mu \mathrm{M})$ under hypoxia for $8 \mathrm{~h}$. Immunoblotting was applied to detect the levels of indicated proteins. HIF-1 $\alpha$, hypoxia-inducible factor $1 \alpha$; BNIP3, BCL2/adenovirus E1B 19 kDa protein-interacting protein 3; siRNA, small interfering RNA; GEM, gemcitabine; LC3, light chain 3.

Annexin V-FITC/PI staining followed by flow cytometry was subsequently used to analyze apoptosis. The apoptotic percentages of T24 cells treated with gemcitabine under normoxic conditions were higher than those observed under hypoxic conditions. However, the apoptotic percentages of T24 cells treated with gemcitabine under hypoxic conditions was significantly augmented following the suppression of autophagy with 3MA (Fig. 5B and C). A similar apoptosis enhancement was also detected using immunoblotting. Increased cleaved caspase-3 and cleavage of PARP were noted when cells were co-treated with gemcitabine and 3MA under hypoxic conditions (Fig. 5D). These results suggested that the resistance of bladder cancer cells to gemcitabine may be attributed to hypoxia-mediated protective autophagy. Decreasing autophagy may therefore enhance gemcitabine-induced apoptosis.

HIF-1 $\alpha / B N I P 3 / B e c l i n 1$ pathway mediates hypoxia-activated autophagy in bladder cancer cells. To investigate the mechanism through which hypoxia induced autophagy in bladder cancer cells, the study determined whether HIF-1 $\alpha$ was involved in the process of hypoxia-induced autophagy. To verify the hypothesis, the activation of HIF-1 $\alpha$ and its downstream target proteins (BNIP3 and Beclin1) was first examined in bladder cancer cells under hypoxic conditions. HIF-1 $\alpha$, BNIP3 and Beclin1 levels were increased in a time-dependent manner under hypoxic conditions in T24 cells (Fig. 6A). A specific siRNA targeting HIF-1 $\alpha$ was then transfected into bladder cancer cell lines to knockdown HIF-1 $\alpha$ expression. As expected, the inhibition of HIF-1 $\alpha$ expression decreased the levels of the HIF-1 $\alpha$ downstream target proteins BNIP3 and Beclin1, and also reduced hypoxia-induced autophagy (Fig. 6B). The results mentioned above suggested that hypoxia promoted gemcitabine-induced autophagy. Therefore, the present study further investigated whether HIF-1 $\alpha$ was involved in the gemcitabine-induced autophagy increase in bladder cancer cells. Following the downregulation of HIF-1 $\alpha$, gemcitabine failed to induce more LC3-II accumulation under hypoxic conditions (Fig. 6C). This demonstrated that HIF-1 $\alpha$ was a main regulator of hypoxia-induced autophagy.

HIF-1a knockdown promoted gemcitabine-induced apoptosis in bladder cancer under hypoxic conditions. To further investigate the role of HIF-1 $\alpha$ in gemcitabine resistance, the effects of HIF-1 $\alpha$ knockdown on gemcitabine-induced cytotoxicity were examined. The knockdown of HIF-1 $\alpha$ almost completely eliminated the resistance of bladder cancer cells to gemcitabine under hypoxic conditions. In addition, HIF-1 $\alpha$ knockdown strongly inhibited cell viability compared with that in cells treated with gemcitabine under hypoxic conditions alone (Fig. 7A). Annexin V-FITC/PI staining followed by flow cytometry was used to measure apoptosis levels. The data revealed that gemcitabine-induced apoptosis increased significantly compared with that in the hypoxia group (Fig. 7B and $\mathrm{C}$ ). However, gemcitabine-induced apoptosis of bladder cancer cells was reversed following the inhibition of HIF-1 $\alpha$ expression. Consistently, immunoblotting demonstrated the same apoptotic changes. Knockdown of HIF-1 $\alpha$ was observed to enhance the activation of caspase-3 and PARP cleavage (Fig. 7D). These results suggested that hypoxia-induced protective autophagy mediated by HIF-1 $\alpha$ may lead to bladder cancer cell resistance to gemcitabine.

\section{Discussion}

The current study provided evidence that HIF- $1 \alpha$-mediated hypoxia-induced autophagy was critical to gemcitabine resistance. Autophagy was evidently induced when T24 cells were treated with hypoxia or gemcitabine alone, and it was noted that hypoxia significantly enhanced autophagy when bladder cancer cells were exposed to gemcitabine. In addition, HIF-1 $\alpha$ expression was increased in bladder cancer tissues and bladder cancer cells treated with hypoxia. HIF-1 $\alpha$ siRNA or treatment with the autophagy inhibitor 3MA inhibited the hypoxia- 
A

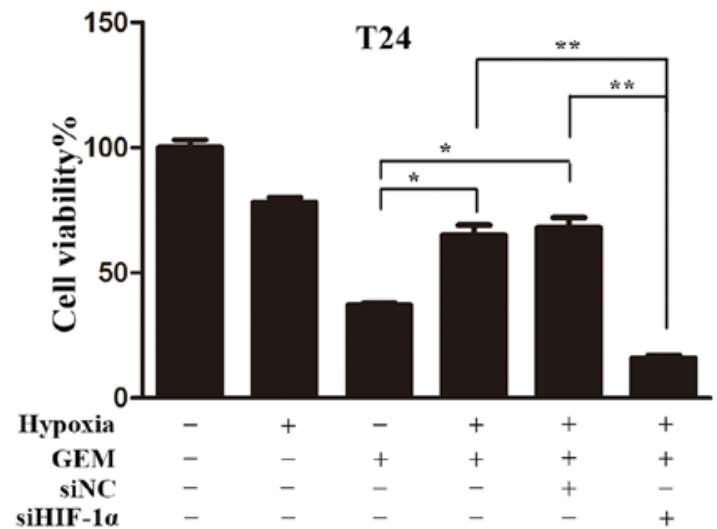

B

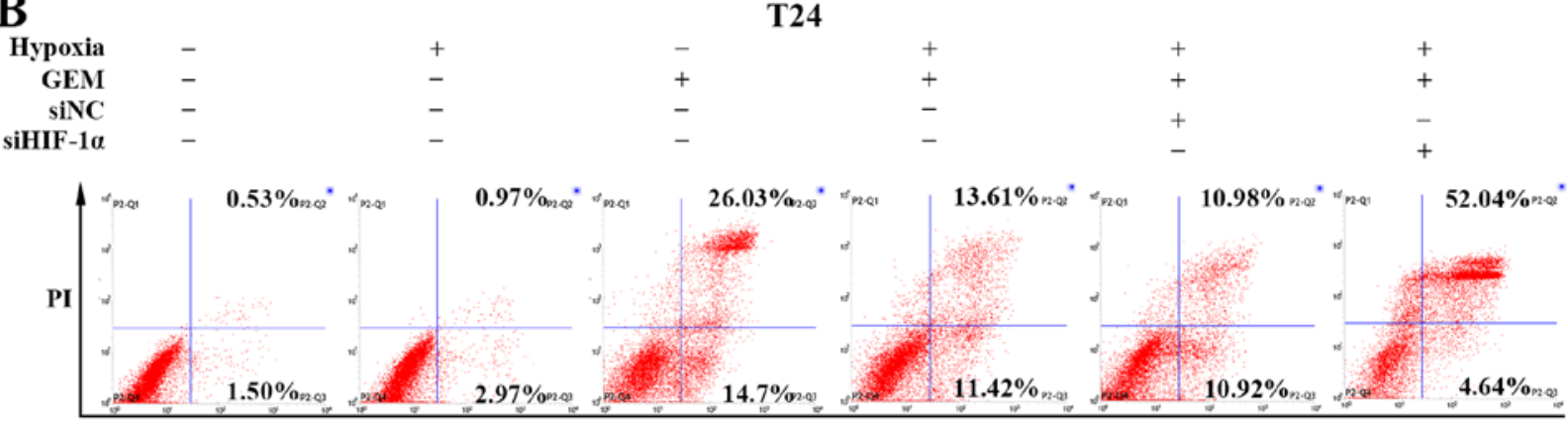

C

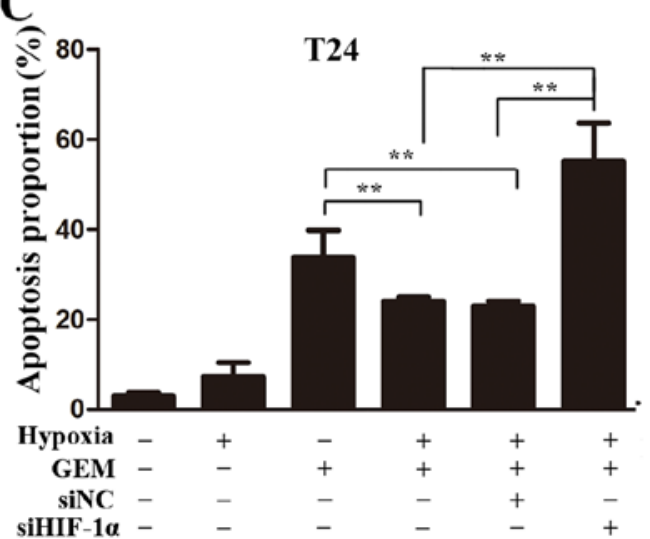

D
T24

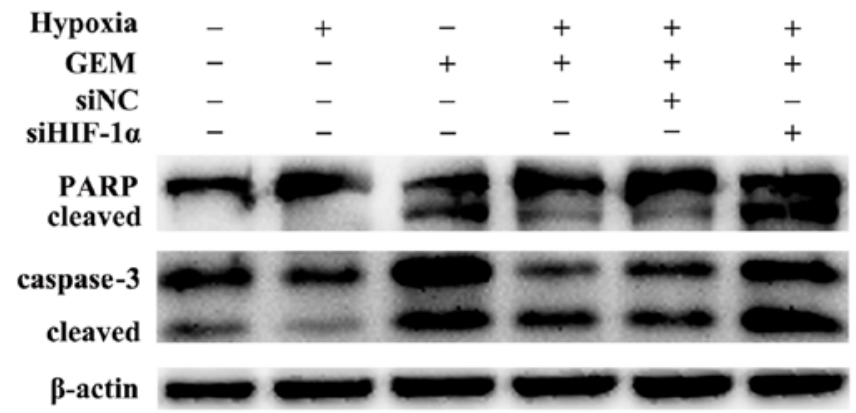

Figure 7. Knockdown of HIF-1 $\alpha$ enhanced GEM-induced apoptosis. T2 4 cells were transfected with HIF-1 $\alpha$ siRNA or control siRNA for 48 h, followed by treatment with GEM $(5 \mu \mathrm{M})$ under hypoxia for $24 \mathrm{~h}$. (A) Cell viability was measured by cell counting kit- 8 assay. (B) Flow cytometry was used to measure the apoptotic cell percentages. (C) Early apoptosis (Annexin $\mathrm{V}^{+} / \mathrm{PI}^{-}$) and late-stage apoptosis (Annexin $\mathrm{V}^{+} / \mathrm{PI}^{+}$) were calculated, and are shown in the histograms. (D) Cleaved caspase-3 and PARP were detected by immunoblotting. Data are shown as the mean \pm standard deviation from three independent experiments. ${ }^{* *} \mathrm{P}<0.01$ and ${ }^{*} \mathrm{P}<0.05$. HIF-1 $\alpha$, hypoxia-inducible factor $1 \alpha$; GEM, gemcitabine; siRNA, small interfering RNA; PARP, poly(ADP-ribose) polymerase.

activated autophagy and augmented the gemcitabine-induced apoptosis. Furthermore, HIF-1 $\alpha$ strongly elevated BNIP3 and Beclin1 expression levels. Suppression of HIF-1 $\alpha$ was observed to downregulate the expression levels of BNIP3 and Beclin1, suggesting that the HIF-1 $\alpha / \mathrm{BNIP} 3 / \mathrm{Beclin} 1$ signaling cascade pathway was involved in hypoxia-induced autophagy. Taken together, the present study results suggested that targeting autophagy or HIF-1 $\alpha$ increased the chemosensitivity of bladder cancer cells to gemcitabine.

Chemotherapy is an important treatment for advanced bladder cancer; however, resistance to anti-cancer drugs has been a key obstacle to further extending the survival of patients. Recent studies have suggested that the local microenvironment of the tumor (particularly hypoxia) serves a significant part in the induction of tumor resistance $(21,22)$.
Consistent with the findings of previous studies $(20,21,23)$, the present study found that T24 cells treated with gemcitabine under hypoxic conditions exhibited significant treatment resistance compared with that observed under normoxic conditions. The underlying mechanisms of hypoxia-induced chemoresistance are complex, and include multiple drugresistant gene expression, reduced reactive oxygen species (ROS) levels, cell cycle arrest, gene mutations and drug concentration decreases $(8,24-27)$. Furthermore, autophagy activation is another possible mechanism protecting cells from chemotherapeutic drug-induced apoptosis. It is generally accepted that autophagy is a mechanism of cellular defense and stress regulation. In the absence of oxygen or nutritional deficiencies, autophagy selectively removes some of the damaged organelles, including mitochondria, endoplasmic 
reticulum and peroxisomes, to maintain cell survival $(28,29)$. In recent years, it was demonstrated that autophagy was also a protective mechanism in the process of chemoresistance under hypoxic conditions in a variety of tumor cells, such as in liver, lung and colon cancer (30-32). However, whether autophagy is involved in the process of bladder cancer cell resistance to chemotherapy under a hypoxic microenvironment is not clear.

In the present study, similarly to previous studies $(6,18,33)$, it was observed that autophagy was activated when bladder cancer cells were exposed to hypoxia, as well as to gemcitabine. Notably, autophagy was enhanced when the cells were exposed to hypoxia and gemcitabine simultaneously, including increased GFP-LC3 puncta, enhanced formation of autophagic vacuoles and upregulation of LC3-II levels. However, when autophagy was blocked by treatment with 3MA, a commonly used inhibitor of autophagy that inhibits the conversion of LC3-I to LC3-II by inhibiting PI3K (34), the hypoxia-induced autophagy was significantly reduced. Furthermore, it has been demonstrated that 3MA combined with chemotherapy drugs under hypoxic conditions can effectively promote tumor cell apoptosis $(14,15)$. As expected, 3MA reversed the chemosensitivity of bladder cancer cells to gemcitabine under hypoxic conditions, which was demonstrated by the enhanced cell proliferation inhibition, increased apoptotic cell percentage, and increased caspase-3 activation and PARP cleavage.

The adaptation of cells to hypoxia mainly depends on changes in gene transcription levels (35). HIF-1 $\alpha$ is an oxygenregulating subunit of HIF-1, a hypoxia-dependent protein, that serves a major hypoxic regulatory role and determines HIF-1 activity. The $\alpha$ subunit of HIF- $1 \alpha$ is easily degraded at high concentrations of oxygen. After stably and rapidly accumulating in the nucleus under hypoxic conditions, HIF- $1 \alpha$ binds with HIF- $1 \beta$ to activate the HIF-1 heterodimeric complex, which promotes the expression of hypoxia target genes through combining with the hypoxia response element $(33,36,37)$. Studies have revealed that the associations between hypoxia and autophagy are mainly through HIF-1regulated expression of downstream target proteins BNIP3 and BNIP3L, which release Beclin1 to activate autophagy by disrupting the interaction between Beclin1 and Bcl-2/Bcl-xL complexes $(33,38-40)$. Another study has suggested that hypoxia-induced cancer cell autophagy was an HIF-1 independent pathway. Hypoxia activated autophagy through activating AMPK-mTOR signaling (41-43). In addition, the unfolded protein region generated by the endoplasmic reticulum under hypoxic stimulation activated transcription factor 4 to induce autophagy (44-46). The present study demonstrated that the expression of HIF- $1 \alpha$ in bladder cancer cells under hypoxic conditions was accompanied with the activation of the downstream proteins BNIP3 and Beclin1. Suppressing HIF- $1 \alpha$ reduced the expression of BNIP3 and Beclin1, and the autophagy activation, suggesting that the HIF-1 $\alpha /$ BNIP3/ Beclin1 signaling cascade was involved in hypoxia-induced autophagy. Furthermore, when HIF-1 $\alpha$ was upregulated under hypoxic conditions, the gemcitabine-induced apoptosis was significantly reduced. However, when HIF-1 $\alpha$ was suppressed, gemcitabine-induced apoptosis was significantly increased. These results indicated that HIF- $1 \alpha$ inhibited the gemcitabine apoptotic cytotoxicity in bladder cancer cells by mediating autophagy.
In conclusion, the results of the current study revealed that resistance to gemcitabine in bladder cancer cells under hypoxic conditions was due to elevated levels of autophagy, which was regulated by HIF- $1 \alpha$-associated signaling pathways. Therefore, the hypoxia-autophagy pathway may be a target for enhancing the effectiveness of gemcitabine chemotherapy in bladder cancer patients.

\section{Acknowledgements}

Not applicable.

\section{Funding}

This study was supported by funding from Natural Science Foundation of China (grant no. 81372758), the Natural Science Fund Project of Chongqing (grant no. cstc2013jcyjA10058), and the Innovative Project of Science Research for Postgraduate of Chongqing Municipal Education Committee (CYS15141).

\section{Availability of data and materials}

The datasets used and/or analyzed during the current study are available from the corresponding author on reasonable request.

\section{Authors' contributions}

WH designed the research; XY performed the experiments; XY, HY, YZ, HT, QW, XL and YZ analyzed the data; XY wrote the manuscript. All authors have read and approved the final manuscript.

\section{Ethics approval and consent to participate}

The study was approved by the Ethics Committee of the First Affiliated Hospital of Chongqing Medical University, and written informed consent was obtained from the patients or the patients' families.

\section{Consent for publication}

Not applicable.

\section{Competing interests}

The authors declare that they have no competing interests.

\section{References}

1. Siegel RL, Miller KD and Jemal A: Cancer Statistics, 2017. CA Cancer J Clin 67: 7-30, 2017.

2. Ghoneim MA, Abdel-Latif M, el-Mekresh M, Abol-Enein H, Mosbah A, Ashamallah A and el-Baz MA: Radical cystectomy for carcinoma of the bladder: 2,720 consecutive cases 5 years later. J Urol 180: 121-127, 2008.

3. Park JC, Citrin DE, Agarwal PK and Apolo AB: Multimodal management of muscle-invasive bladder cancer. Curr Probl Cancer 38: 80-108, 2014.

4. Geldart T, Chester J, Casbard A, Crabb S, Elliott T, Protheroe A, Huddart RA, Mead G, Barber J, Jones RJ, et al: SUCCINCT: An open-label, single-arm, non-randomised, phase 2 trial of gemcitabine and cisplatin chemotherapy in combination with sunitinib as first-line treatment for patients with advanced urothelial carcinoma. Eur Urol 67: 599-602, 2015. 
5. Toschi L, Finocchiaro G, Bartolini S, Gioia V and Cappuzzo F: Role of gemcitabine in cancer therapy. Future Oncol 1: 7-17, 2005.

6. Huang XL, Zhang H, Yang XY, Dong XY, Xie XY, Yin HB, Gou X, Lin Y and He WY: Activation of a c-Jun N-terminal kinase-mediated autophagy pathway attenuates the anticancer activity of gemcitabine in human bladder cancer cells. Anticancer Drugs 28: 596-602, 2017.

7. Dauer P, Nomura A, Saluja A and Banerjee S: Microenvironment in determining chemo-resistance in pancreatic cancer: Neighborhood matters. Pancreatology 17: 7-12, 2017.

8. Yasuda H: Solid tumor physiology and hypoxia-induced chemo/ radio-resistance: Novel strategy for cancer therapy: Nitric oxide donor as a therapeutic enhancer. Nitric Oxide 19: 205-216, 2008.

9. Frolova O, Samudio I, Benito JM, Jacamo R, Kornblau SM, Markovic A, Schober W, Lu H, Qiu YH, Buglio D, et al: Regulation of HIF-1 $\alpha$ signaling and chemoresistance in acute lymphocytic leukemia under hypoxic conditions of the bone marrow microenvironment. Cancer Biol Ther 13: 858-870, 2012.

10. Qiu Y, Li P and Ji C: Cell death conversion under hypoxic condition in tumor development and therapy. Int J Mol Sci 16: 25536-25551, 2015.

11. Schito L and Semenza GL: Hypoxia-inducible factors: Master regulators of cancer progression. Trends Cancer 2: 758-770, 2016.

12. Song J, Qu Z, Guo X, Zhao Q, Zhao X, Gao L, Sun K, Shen F, Wu M and Wei L: Hypoxia-induced autophagy contributes to the chemoresistance of hepatocellular carcinoma cells. Autophagy 5: 1131-1144, 2009.

13. Mizushima N: Autophagy: Process and function. Genes Dev 21: 2861-2873, 2007.

14. Wu HM, Jiang ZF, Ding PS, Shao LJ and Liu RY: Hypoxiainduced autophagy mediates cisplatin resistance in lung cancer cells. Sci Rep 5: 12291, 2015.

15. Liu XW, Su Y, Zhu H, Cao J, Ding WJ, Zhao YC, He QJ and Yang B: HIF-1 $\alpha$-dependent autophagy protects HeLa cells from fenretinide (4-HPR)-induced apoptosis in hypoxia. Pharmacol Res 62: 416-425, 2010.

16. Helpap B: New WHO classification of urothelial carcinoma of the urinary bladder. Verh Dtsch Ges Pathol 86: 57-66, 2002 (In German).

17. Compérat EM, Burger M, Gontero P, Mostafid AH, Palou J, Rouprêt M, van Rhijn BWG, Shariat SF, Sylvester RJ, Zigeuner R, et al: Grading of urothelial carcinoma and the new 'World Health Organisation Classification of Tumours of the Urinary System and Male Genital Organs 2016'. Eur Urol Focus S2405-4569(18)30004-X, 2018.

18. Lee JG, Shin JH, Shim HS, Lee CY, Kim DJ, Kim YS and Chung KY: Autophagy contributes to the chemo-resistance of non-small cell lung cancer in hypoxic conditions. Respir Res 16: $138,2015$.

19. Huang Z, Zhong Z, Zhang L, Wang X, Xu R, Zhu L, Wang Z, Hu $S$ and Zhao X: Down-regulation of HMGB1 expression by shRNA constructs inhibits the bioactivity of urothelial carcinoma cell lines via the NF- $\mathrm{BB}$ pathway. Sci Rep 5: 12807, 2015.

20. He X, Wang J, Wei W, Shi M, Xin B, Zhang T and Shen X: Hypoxia regulates ABCG2 activity through the activivation of ERK1/2/HIF-1 $\alpha$ and contributes to chemoresistance in pancreatic cancer cells. Cancer Biol Ther 17: 188-198, 2016.

21. Ko YH, Cho Y-S, Won HS, Jeon EK and Hong YS: Possible role of autophagy inhibition in hypoxia-induced chemoresistance of pancreatic cancer cells. J Clin Oncol 30 (Suppl. 4): 224, 2012.

22. Ma Q, Zhang Y, Liu T, Jiang K, Wen Y, Fan Q and Qiu X: Hypoxia promotes chemotherapy resistance by down-regulating SKA1 gene expression in human osteosarcoma. Cancer Biol Ther 18: 177-185, 2017.

23. Guo Q and Qin W: DKK3 blocked translocation of $\beta$-catenin/ EMT induced by hypoxia and improved gemcitabine therapeutic effect in pancreatic cancer Bxpc-3 cell. J Cell Mol Med 19: 2832-2841, 2015.

24. Guo XL, Li D, Sun K, Wang J, Liu Y, Song JR, Zhao QD, Zhang SS, Deng WJ, Zhao X, et al: Inhibition of autophagy enhances anticancer effects of bevacizumab in hepatocarcinoma. J Mol Med (Berl) 91: 473-483, 2013.

25. Wang Q, Yang Y, Wang L, Zhang PZ and Yu L: Acidic domain is indispensable for MDM2 to negatively regulate the acetylation of p53. Biochem Biophys Res Commun 374: 437-441, 2008.

26. Wartenberg M, Hoffmann E, Schwindt H, Grünheck F, Petros J, Arnold JR, Hescheler J and Sauer H: Reactive oxygen specieslinked regulation of the multidrug resistance transporter P-glycoprotein in Nox-1 overexpressing prostate tumor spheroids. FEBS Lett 579: 4541-4549, 2005.
27. Chen WL, Wang CC, Lin YJ, Wu CP and Hsieh CH: Cycling hypoxia induces chemoresistance through the activation of reactive oxygen species-mediated B-cell lymphoma extra-long pathway in glioblastoma multiforme. J Transl Med 13: 389, 2015.

28. Ravanan P, Srikumar IF and Talwar P: Autophagy: The spotlight for cellular stress responses. Life Sci 188: 53-67, 2017.

29. Yin H, Yang X, Gu W, Liu Y, Li X, Huang X, Zhu X, Tao Y, Gou $X$ and He W: HMGB1-mediated autophagy attenuates gemcitabine-induced apoptosis in bladder cancer cells involving JNK and ERK activation. Oncotarget 8: 71642-71656, 2017.

30. Kabir N and Yong Y: Hypoxia-induced autophagy in hepatocellular carcinoma and anticancer therapy. Natl J Physiol Pharm Pharmacol 7: 771-780, 2017.

31. Notte A, Ninane N, Arnould T and Michiels C: Hypoxia counteracts taxol-induced apoptosis in MDA-MB-231 breast cancer cells: Role of autophagy and JNK activation. Cell Death Dis 4: e638, 2013.

32. Selvakumaran M, Amaravadi RK, Vasilevskaya IA and O'Dwyer PJ: Autophagy inhibition sensitizes colon cancer cells to antiangiogenic and cytotoxic therapy. Clin Cancer Res 19: 2995-3007, 2013

33. Zou YM, Hu GY, Zhao XQ, Lu T, Zhu F, Yu SY and Xiong H: Hypoxia-induced autophagy contributes to radioresistance via c-Jun-mediated Beclin1 expression in lung cancer cells. J Huazhong Univ Sci Technolog Med Sci 34: 761-767, 2014.

34. Vergne I, Roberts E, Elmaoued RA, Tosch V, Delgado MA, Proikas-Cezanne T, Laporte J and Deretic V: Control of autophagy initiation by phosphoinositide 3-phosphatase Jumpy. EMBO J 28: 2244-2258, 2009.

35. Adams JM, Difazio LT, Rolandelli RH, Luján JJ, Haskó G, Csóka B, Selmeczy Z and Németh ZH: HIF-1: A key mediator in hypoxia. Acta Physiol Hung 96: 19-28, 2009.

36. $\mathrm{Li}$ YN, Hu JA and Wang HM: Inhibition of HIF-1 $\alpha$ affects autophagy mediated glycosylation in oral squamous cell carcinoma cells. Dis Markers 2015: 239479, 2015.

37. Harada H, Itasaka S, Kizaka-Kondoh S, Shibuya K, Morinibu A, Shinomiya $\mathrm{K}$ and Hiraoka M: The Akt/mTOR pathway assures the synthesis of HIF-1alpha protein in a glucose- and reoxygenation-dependent manner in irradiated tumors. J Biol Chem 284: 5332-5342, 2009.

38. Hu YL, DeLay M, Jahangiri A, Molinaro AM, Rose SD, Carbonell WS and Aghi MK: Hypoxia-induced autophagy promotes tumor cell survival and adaptation to antiangiogenic treatment in glioblastoma. Cancer Res 72: 1773-1783, 2012.

39. Bellot G, Garcia-Medina R, Gounon P, Chiche J, Roux D, Pouysségur J and Mazure NM: Hypoxia-induced autophagy is mediated through hypoxia-inducible factor induction of BNIP3 and BNIP3L via their BH3 domains. Mol Cell Biol 29: 2570-2581, 2009

40. Huang HY, Wang WC, Lin PY, Huang CP, Chen CY and Chen YK: The roles of autophagy and hypoxia in human inflammatory periapical lesions. Int Endod J 51 (Suppl 2): e125-e145, 2018.

41. Kim J, Kundu M, Viollet B and Guan KL: AMPK and mTOR regulate autophagy through direct phosphorylation of Ulk1. Nat Cell Biol 13: 132-141, 2011.

42. Jin Y, Bai Y, Ni H, Qiang L, Ye L, Shan Y and Zhou M: Activation of autophagy through calcium-dependent AMPK/mTOR and PKC $\theta$ pathway causes activation of rat hepatic stellate cells under hypoxic stress. FEBS Lett 590: 672-682, 2016.

43. Liu H, Qiu H, Xiao Q and Le W: Chronic hypoxia-induced autophagy aggravates the neuropathology of Alzheimer's disease through AMPK-mTOR signaling in the APPSwe/PS1dE9 mouse model. J Alzheimers Dis 48: 1019-1032, 2015.

44. Fang Y, Tan J and Zhang Q: Signaling pathways and mechanisms of hypoxia-induced autophagy in the animal cells. Cell Biol Int 39: 891-898, 2015.

45. Pike LR, Singleton DC, Buffa F, Abramczyk O, Phadwal K, Li JL, Simon AK, Murray JT and Harris AL: Transcriptional up-regulation of ULK1 by ATF4 contributes to cancer cell survival. Biochem J 449: 389-400, 2013.

46. Rzymski T, Milani M, Pike L, Buffa F, Mellor HR, Winchester L, Pires I, Hammond E, Ragoussis I and Harris AL: Regulation of autophagy by ATF4 in response to severe hypoxia. Oncogene 29: 4424-4435, 2010. 